\title{
Comunicação e Escuta
}

Jacques Alkalai Wainberg

\section{Resumo}

Este é um estudo teórico e empírico sobre a escuta, uma habilidade que implica atributos cognitivos e emocionais do ouvinte. A resenha teórica mostra seus principais elementos e desafios, os quais dizem respeito a todo tipo de interlocução, como a conversação e a sintonia à mídia, por exemplo. Aplica-se aqui o questionário Hurier Listening Profile a 80 universitários. 0s dados levantados mostram que a escuta é 0 atributo cognitivo que apresenta a maior dificuldade entre os seis envolvidos na audição. 0 estudo destaca também o papel das emoções na estimulação da atenção da audiência.

\section{Palavras-Chave}

Escuta. Emoção. Cognição.

\section{Jacques Alkalai Wainberg | jacqalwa@pucrs.br}

Doutor em Ciências da Comunicação pela Universidade de São Paulo - USP, Brasil. Pós-Doutor pela Universidade do Texas, EUA, e pela Universidade de Tel Aviv, Israel. Prof. do Programa de Pós-Graduação em Comunicação Social da Pontifícia Universidade Católica do Rio Grande do Sul - PUCRS, Brasil.
Na sociedade moderna, muitos falam e poucos ouvem. Essa queixa é usual entre os que lamentam a crise do diálogo e da conversação (TURKLE, 2016). Para eles, a escuta é um sentido depreciado ${ }^{1}$, especialmente no campo da política (DOBSON, 2014; p. 3; BICKFORD, 1996), em que predominam o embate, o monólogo e 0 desejo de os atores vencerem e se sobreporem aos opositores (WAINBERG, 2010). Nessas disputas, costuma-se mencionar 0 direito à liberdade de expressão, mas não o dever de escuta, condição necessária à deliberação.

Como não é possível baixar uma lei a respeito, a escuta é habilidade que acaba dependendo dos costumes e da etiqueta social, simplesmente. 0s 10 melhores hábitos de escuta a serem ensinados foram desenvolvidos e popularizados por Ralph G. Nichols (NICHOLS, julho de 1957). Suas recomendações, uma das primeiras entre as inúmeras que se propagaram desde então, ficaram conhecidas como Nichols $10 .{ }^{2}$ 
“Nichols 10": Hábitos para uma pessoa escutar bem. Ralph G. Nichols. 1957

\begin{tabular}{|c|l|}
\hline 1 & Encontrar uma área de interesse. \\
\hline 2 & Julgar o conteúdo, não o falante. \\
\hline 3 & 0 ouvinte deve se conter até entender plenamente o que está sendo dito pelo falante. \\
\hline 4 & Prestar atenção na ideia principal. \\
\hline 5 & Ser flexível. \\
\hline 6 & Esforçar-se para ouvir. \\
\hline 7 & Esforçar-se para não se distrair. \\
\hline 8 & Exercitar a mente na audição. \\
\hline 9 & Manter a mente aberta. \\
\hline 10 & Utilizar a energia auditiva para focar o pensamento e refletir sobre 0 que está sendo dito. \\
\hline
\end{tabular}

Fonte: http://www.listen.org/Resources/Documents/Nichols10PartSkill.pdf

$\mathrm{Na}$ ciência política, predomina 0 desinteresse pelo tema (DOBSON, 2014). Esta falta de legitimidade existe também no campo da comunicação (PELL et al., 2011, p. 835; BERD, D. \& BODIE, G. D., 2014), apesar de a escuta ser tópico aplicado e relevante a inúmeras ocorrências do dia a dia. Exemplo é a comunicação entre os pilotos e as torres de controle dos aeroportos, um tema grave para a segurança da aviação; a interação entre professores e alunos na sala de aula; 0 papel dos mediadores que almejam facilitar a relação entre as partes envolvidas num conflito; a interação entre negociadores; o novo fenômeno organizacional das ouvidorias e hotlines; a habilidade de os médicos ouvirem bem para diagnosticarem bem; os pais que precisam escutar os filhos e vice-versa; as conversas telefônicas, algo que depende da qualidade da audição, e 0 caso dos terapeutas que vivem profissionalmente da escuta. Por fim, há ainda o fenômeno das sintonias. Milhares escutam o rádio, a locução das notícias e o ritmo da música.

Surpreende, portanto, a relativa estranheza dos estudos de comunicação ao assunto. 0 tópico é raro nessa área também no Brasil ${ }^{3}$. Quando ocorrem trabalhos, são aproximativos e descritivos, de tom filosófico. Não são empíricos,

https://www.youtube.com/watch?v=ws5TRAleqs4

http://www.listen.org/Resources/Documents/Nichols10PartSkill.pdf

Revisão realizada pelo autor constatou que não há, nas revistas acadêmicas da área, textos cuja palavra-chave remeta à escuta. 0 usual é encontrar estudos sobre sintonia, áudio, ouvintes, discurso, audiência, entre outros tópicos similares. 0 papel da escuta na comunicação é muito pouco considerado. 0 tema é bem mais frequente nos estudos de psiquiatria e psicanálise, educação, psicologia, enfermagem, fonoaudiologia, religião, música, musicoterapia e áreas correlatas. 
e os resultados apresentados no país e no exterior são, com frequência, considerados ambíguos (BOSTROM \& BRYANT, 1980). 0 desejo em afirmar e dar legitimidade a essa tradição de investigação é 0 que explica 0 esforço feito ao longo do tempo para desenvolver testes e experimentos laboratoriais sobre esse tipo de recepção. Tais estudos avaliam 0 contato visual entre falantes e ouvintes durante a conversação, ofeedback oferecido por um e outro à interação, a recordação pelo ouvinte do que foi dito pelo falante e o tipo de audição, algo que depende do contexto, entre outros tópicos de investigaçã $0^{4}$.

Essa propriedade pragmática, a que considera 0 texto em seu contexto, é usualmente assinalada na análise dos discursos, embora ela seja útil igualmente para explicar o comportamento do audiente. Afinal, a pessoa escuta de várias maneiras - na mercearia, na conversa com seu superior, com o médico e na companhia de seus amigos. A audição ativa e empática é a que surge, por exemplo, na relação de intimidade da pessoa com 0 seu interlocutor próximo. 0 ambiente terapêutico almeja criar relação similar entre 0 analista e o paciente (FROMM, 2013). É o que agora se denomina de escuta intensiva. Ouvir música implica a escuta apreciativa. 0 explorador que desbrava um novo ambiente faz uma escuta discriminativa e profunda das novas redondezas
(HUTCHBY, 2005; LIBOW \& DOTY, 1976; BODIE, 2016) ${ }^{5}$. Surgem, por decorrência, os estilos de escuta. 0 desejo de conexão é denominado de escuta relacional. Quando o ouvinte foca no conteúdo da fala do orador, a escuta passa a ser chamada de analítica. Já a escuta crítica refere-se ao ouvinte que examina a precisão e a consistência do pronunciamento do orador (GEARHART, DENHAM \& BODIE, 2014).

Escutar implica atividades complexas, tais como filtrar, processar, registrar, dar sentido e significado ao que se ouve (GLENN, 1989) ${ }^{6}$. São etapas que revelam o grau de engajamento do ouvinte com o que lhe chega aos ouvidos. Embora a pessoa ouça muita coisa ao mesmo tempo, ela não consegue escutar tudo; por isso mesmo, a pessoa que decide escutar avalia e seleciona com algum critério esses estímulos. Para tanto, ela leva em consideração a relevância dos sons, algo que produz certo grau de excitação e que lhe predispõe alocar certo volume de energia para sua decifração (KAHNEMAN, 1973). É o que ensina a Teoria ou Efeito Coquetel, metáfora utilizada para mostrar como, num ambiente ruidoso, a pessoa é capaz, assim mesmo, de focar sua atenção auditiva em algo que lhe interessa sobremaneira (CHERRY, 1953; TREISMAN, 1969; BROADBENT, 1954). Isso depende também da importância semântica dos sons para o ouvinte

Ver p. ex. http://www.queendom.com/tests/access_page/index.htm?idRegTest=3131

http://cultureofempathy.com/Projects/Empathy-Movement/References/Reflective-Listening.htm 
(TREISMAN, 1969; DEUTSCH \& DEUTSCH, 1969); ou seja, a pessoa sempre exclui os inputs auditivos (e outros) que lhe são irrelevantes em dado momento (LIMA, 2015).

0 acrônimo inglês SIER (Sensing, Interpreting, Evaluating e Responding) é um modelo de audição (ativa e reflexiva) concebido por Steil, Watson \& Barker em 1947. Ele gera em português 0 acrônimo PIAR (Perceber, Interpretar, Avaliar e Responder). 0 PIAR indica o processo através do qual a pessoa internaliza uma mensagem ouvida. Segundo os autores, o ouvinte que escuta recorda, após a interlocução, $50 \%$ do que ouve e, dois dias depois, somente $25 \%$. Outras fontes dizem que esse indicador é de 10\% logo após a conversa se encerrar (STAFFORD, BURGGRAF, \& SHARKEY, 1987; STAFRORD \& DALY, 1984). 0 fato é que, à medida que 0 tempo passa, as pessoas recordam mais a essência do diálogo e menos palavras e frases específicas (MILLER \& de WINSTANLEY, 2002; MILLER et al, 1996).

Modelo PIAR/SIER de audição ativa (Steil, Watson \& Barker, 1947)

PERCEBER - INTERPRETAR - AVALIAR - RESPONDER

Outro acrônimo inglês, o HURIER (Hearing, Understanding, Remembering, Interpreting,

Evaluating, Responding), refere-se ao único modelo de audição validado empiricamente (BROWNELL, 1995). Em português, surge o acrônimo equivalente
ECRIAR (Escutar, Compreender, Recordar, Interpretar, Avaliar e Responder).

Modelo ECRIAR/HURRIER de audição (BROWNELL, 1995)

\section{ESCUTAR - COMPREENDER - RECORDAR -} INTERPRETAR - AVALIAR - RESPONDER

Ouvir, em condições normais, é uma habilidade física da qual todas as demais dependem. É um atributo orgânico e inato. É a capacidade física que permite a uma pessoa capturar os sons e os ruídos do ambiente. Nessa etapa, ela foca a atenção num estímulo determinado; depois, o esforço de escutar permanece fixado no alvo sem se dispersar. Compreender, a segunda etapa, envolve não só a decodificação de um signo, mas sua relação com o contexto e com 0 repertório preservado no banco de dados mental da pessoa. Recordar, a terceira etapa, implica 0 arquivamento da nova informação para futuro uso. Dotar de significado certa mensagem é interpretar, a quarta etapa. Naturalmente, quem escuta também avalia o falante, sua credibilidade e sua intenção, por exemplo; essa é a quinta etapa. A que segue, a sexta e última, é oferecer ao falante uma resposta. Então, finalmente, os papéis se invertem. É a interlocução.

Existem inúmeros outros modelos ainda. Seus elementos mais frequentes são a atenção dada pelo ouvinte ao estímulo, sua resposta verbal e não verbal ao interlocutor, o processamento cognitivo da audição (atenção, compreensão, recepção e 
interpretação) e o processamento comportamental (afetivo) do ouvinte. A definição oferecida pela International Listening Association é uma das mais populares? ${ }^{7}$ Ela diz que escutar é "0 processo de receber, construir significado e responder às mensagens verbais e não verbais" (EMMERT, 1996, p. 2-3). Michael Purdy expandiu a formulação: 0 autor diz que ouvir "é o processo ativo e dinâmico de atentar, perceber, interpretar, recordar e responder às necessidades manifestadas (de forma verbal e não verbal), às preocupações e informação oferecidas por outros seres humanos" (1996, p.8). Flynn, Valikoski e Grau (2008, p. 143) argumentam que escutar envolve a capacidade de ouvir e o processamento cognitivo derivado (a habilidade de seletivamente perceber, interpretar, compreender, construir sentido, reagir, recordar e analisar o que foi ouvido). Wolvin e Coakley (1993) afirmam existirem hoje 13 perspectivas teóricas a respeito desse tema.

Uma nova e significativa contribuição ao tema foi oferecida por Janusik $(2004,2007)$. A autora desenvolveu um instrumento para medir a capacidade conversacional da pessoa
(CLS - Conversational Listening Span). 0

$C L S$ determina o número de itens que alguém pode processar e responder no contexto de um diálogo. A propósito, cabe lembrar que pessoas compulsivas, com síndrome do pânico, com ruminação suicida e déficit de atenção escutam menos. Essa habilidade varia igualmente entre os homens e as mulheres (COLLINS, 2006 citado por ROEBUCK, 2015). Variação similar também acontece entre as diferentes culturas (WOLVIN, 1987 citado por ROEBUCK, 2014, 2015).

Ocorre também que algumas pessoas decidem viver no silêncio; simplesmente se afastam do convívio social. Vivem em reclusão, isoladas em contemplação. Estima-se em 7600 o número de monges e monjas trapistas cristãos que habitam 299 mosteiros espalhados pelo mundo nessa condiçã $0^{8}$, inclusive no deserto $0^{9}$. Nesses locais há poucos momentos autorizados para a pessoa falar. É assim que vivem os cartuxos (religiosos seguidores de São Bruno) ${ }^{10}$, as carmelitas (a Ordem do Carmo surgiu no século XI) ${ }^{11}$, os camaldulenses (pertencentes à ordem beneditina fundada no século VI) ${ }^{12}$

http://www.listen.org/

8 cálculo é feito com base nos dados apresentados na Wikipedia.

https://www.youtube.com/watch?v=gu6zwMUek9o

10 Eles estão presentes também em Ivorá, próximo a Santa Maria, no Rio Grande do Sul. http://www.chartreux.org/pt/casas/ medianeira/index.php. Ver também https://www.youtube.com/watch?v=0eHw_sXM6ck

$11 \mathrm{https} / / /$ www.youtube.com/watch?v=Tzla00M18Fo

12 https://www.youtube.com/watch?v=wWlvQ6BwV1g // https://www.youtube.com/watch?v=KTluaAqUl6U 
e também os monges budistas do Tibete ${ }^{13}$. Foi esse estilo de vida o escolhido pelos 300 membros das comunidades esotéricas pitagóricas, as que se propagaram no sul da Itália e ali subsistiram por cerca de 200 anos (KAHN, 2001; p. 25-26; CORNELLI, 2011, p. 230-245). A escuta, no entanto, é uma propriedade cognitiva e psicológica que deve ser desenvolvida e praticada pela pessoa. Trata-se não só de uma operação mental e de um cálculo operacional e racional (frio), mas também de uma disposição afetiva (quente) de a pessoa acolher o interlocutor. Isso é manifestado por meio do esforço do bom ouvinte em decifrar as palavras do falante, em fazer sentido de suas proposições e enunciados e descobrir também o que neles está sendo implicado e, às vezes, interditado. Em suma, a pessoa que ouve escolhe escutar ${ }^{14}$.

Por vezes os efeitos emocionais da interlocução visual e auditiva são sutis. A música ambiente, por exemplo, é tocada geralmente para ser ouvida, mas não para ser escutada. 0 impacto provocado à sensibilidade humana pela trilha musical de um filme dramático é algo notório. Em ambos os casos os estímulos sonoros alcançam a pessoa de forma inconsciente levando-0 a sentir uma emoção e a enxergar a cena de certa maneira.

Emoções como a raiva, a alegria e o orgulho são a via mediante a qual as relações humanas se estabelecem mais rapidamente (JOHNSON,
1998). Elas têm missão vital: no caso do medo, é a sobrevivência e a segurança da pessoa. No caso da tristeza, é o luto. A raiva é uma reação comportamental que visa a proteger certo território; a vergonha deseja a aceitação social; a culpa almeja o respeito; 0 amor almeja produzir conexão (van ALPHEN, 2016). Por isso, as emoções são consideradas como o meio que dispara, rápida e intensamente, 0 engajamento auditivo da pessoa com 0 falante (BOMMELJE, 2013).

A sedução depende dessa capacidade do falante de despertar no ouvinte uma emoção positiva. Trata-se de um esforço persuasivo, o que revela certo cuidado na sua formulação e 0 caráter demagógico deste tipo de manifestação - que tipifica o modelo Don Juan de aproximação. Afinal, cabe lembrar, a demagogia é a arte de "saber conduzir o povo". 0 populismo político também tem essa característica. Seus portavozes são donatários de uma habilidade retórica invejável e de um carisma pessoal comovente, algo que caracteriza as celebridades do showbis e da mídia igualmente.

Em outras palavras, numa conversação, num debate ou noutra ocasião similar, as pessoas demonstram ter dificuldades variadas para seguir e fazer sentido dos inúmeros estímulos que lhes chegam aos sentidos (JUST \& CARPENTER, 1992). Certos fatores emocionais influenciam sua 
decisão de ora considerar e ora desconsiderar os sons que as envolvem (WITKIN, 1980, P. 19). As emoções funcionam como filtros cognitivos; podemos ouvir, mas não escutar 0 que contraria as nossas crenças (NICHOLS \& STEVENS, Setembro de 1957), algo que ocorre com a comunicação dissidente, a que abala o senso comum de uma comunidade. É isso que explica também o vai e vem do dial dos aparelhos de rádio. Os ouvintes inquietos mudam sem cessar a sintonia até encontrar, na programação das emissoras, algo que lhes agrade. Isso ocorre porque a principal característica dessa mídia é oferecer ao público (boa) companhia. No caso brasileiro, 34\% das pessoas que escutam rádio também realizam alguma tarefa doméstica e $33 \%$ o fazem enquanto se alimentam. Outro benefício emocional desta audiência é 0 fato de que o rádio aglutina a sociedade e ajuda a pessoa formar redes sociais (CURCI \& BELLELLI, 2004; p. 881). 0 conteúdo desse canal serve de pano de fundo à conversação interpessoal (26\%) SECOM, 2015). 0 rádio também ocupa 0 tempo livre das pessoas (30\%), informa-as (63\%) e as entretém (62\%). Os dados brasileiros mostram que $52 \%$ dos ouvintes escutam rádio em casa, $15 \%$ no carro, $10 \%$ no trabalho e $6 \%$ no trajeto enquanto se movimentam ${ }^{15}$. Fontes de outros países referendam aproximadamente estes indicadores ${ }^{16}$.

0 poder da voz para 0 audiente é igualmente relevante nos casos do documentário e do telejornalismo. Em ambos o narrador comanda de forma imperceptível o olhar do telespectador. Exemplo disso é a cobertura da II Guerra Mundial realizada pelos cinejornais ${ }^{17}$. 0 que era dito pelo locutor, popularmente chamado e conhecido como A Voz de Deus, induzia o público do cinema a interpretar de certa maneira as imagens captadas pelos cinegrafistas (WAINBERG, 1992). Naturalmente, esse efeito era reforçado pelo fundo musical dramático que acompanhava as cenas das batalhas (muitas delas encenadas no estilo dos docudramas $\left.{ }^{18}\right)$, os navios em alto mar e as tropas aliadas marchando em direção ao inimigo nazista.

Isso explica também porque, agora, o Euronews optou por silenciar os episódios rotulados de No Comment de seu telejornal ${ }^{19}$. Predomina, na maioria dessas reportagens, o som verité, o que é captado no ambiente sem qualquer edição. Nesse caso, a ausência do relato oral do repórter evita o condicionamento do olhar do telespectador. 0

15 Kantar Ibope Meida. In http://aerp.org.br/portal/quem-ouve-radio/

16 http://www.radioworld.com/news-and-business/0002/why-do-listeners-listen/339639 http://www.radioitsalovething.com.au/Why-Radio.aspx http://www.dollywagon.com/2011/01/why-do-people-still-listen-to-radio-is-it-a-social-network-thing/

17 https://www.youtube.com/watch?v=gkaGKSTYV4I

18 Drama documentário; estilo de documentário que apresenta de forma dramática a reconstituição de fatos, utilizando atores para isso. 
noticiário diz com clareza que o propósito desse tipo de cobertura é oferecer ao telespectador a oportunidade de ele tirar "suas próprias conclusões". Algo similar, mas oposto, acontece com a legenda de uma fotografia. Ela enquadra a visão do leitor e condiciona sua interpretação da cena. Uma imagem vale mil palavras somente quando elas estão, efetivamente, ausentes.

Cabe concluir, portanto que os atributos da audição e da visão (MENEZES, 2008), os físicos e os psicológicos, estão fortemente interligados. A deficiência de um deles é compensada pelo aperfeiçoamento de outro. Isso é chamado de modalidade cruzada. Ver implica ouvir (2-1) e vice-versa (1-2), enquanto que enxergar implica escutar (3-4) e vice-versa (4-3). Dito de outra maneira, a pessoa que vê sem enxergar ouve, mas não escuta (2 34 ). Da mesma forma, a pessoa que simplesmente ouve e não escuta vê, mas não enxerga $(144$ ).

\section{VISÃO E AUDIÇÃO - modalidade cruzada}

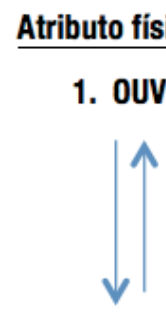

2. VER Atributo psicológico 3.ENXERGAR

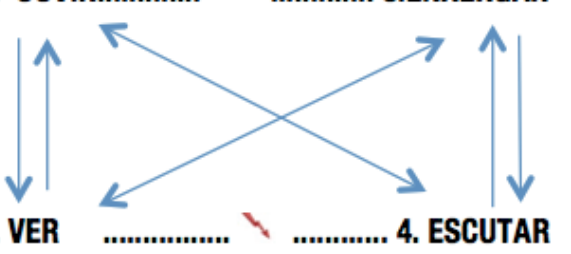

Algo parecido acontecia nas sociedades orais.

Nelas, as narrativas de encantamento eram o meio através do qual as novas gerações eram educadas. As

histórias contadas produziam nos ouvintes imagens

McGill de Montreal mostra iss $0^{20}$. Usualmente, as pessoas falam seis sílabas por segundo. 0 cego, no entanto, pode ouvir até 25 sílabas por segundo. Nesse experimento, um programa de computador foi utilizado para acelerar uma frase. Ela se tornou incompreensível para os ouvintes com visão normal, mas era discernível para os cegos. 0 inverso também ocorre. Os deficientes auditivos enxergam melhor. Pesquisadores da Universidade de Sheffield verificaram que os neurônios da retina dos surdos são distribuídos de forma diferente. Seu campo de visão periférica é maior. Há também um alargamento de uma área do nervo ótico.

Isso significa que os surdos têm mais neurônios transmitindo informações visuais (CODINA et.al, 2017). Experimentos realizados por Amir Amedi, um neurocientista da Universidade Hebraica de Jerusalém, mostram algo excepcional: a pessoa pode enxergar escutando sem ver (3-4 42$)$. $0 \mathrm{u}$ seja, um deficiente visual pode enxergar imagens ao escutar uma sequência de sons que lhe chega biônica e artificialmente ao cérebro ${ }^{21}$.

20 Montreal Neurological Institute / McGill University. "The Blind Really Do Hear Better." ScienceDaily. ScienceDaily, 23 July 2004. <www.sciencedaily.com/releases/2004/07/040723093712.htm>. 
mentais fascinantes, algo que ocorre também no caso das crianças que escutam contos de ninar. Não raro, encantadas, elas pedem aos pais a repetição da mesma fantasia antes de 0 sono as alcançar.

A emoção, por vezes, surge devido à eventual contradição entre o que se diz (o conteúdo semântico do pronunciamento) e como se diz algo (o efeito prosódico, ou seja, a entonação, o ritmo e 0 acento da linguagem falada, o cantarolar de um discurso). É 0 que ocorre no caso da ironia. Nesse caso, o falante dá a impressão de que não está falando sério. Quando as pistas semânticas e prosódicas são congruentes, a emoção gerada no ouvinte é reforçada (GRANDJEAN \& SCHERER, 2006; WILSON \& WHARTON, 2006). A propósito, cabe salientar que os efeitos semânticos não podem ser empiricamente avaliados na ausência completa de pistas prosódicas (PELL et. all, 2011, p. 835), muito embora os primeiros sejam aparentemente mais relevantes que os segundos no transporte das emoções desde 0 falante ao ouvinte (MORTON \& TREHUB, 2010).

Em média 0 ser humano fala e ouve confortavelmente entre 150 e 160 palavras por minuto (WILLIAMS, 1998). Os leiloeiros vão além e gritam até 250 palavras. Alguns debatedores podem chegar a 500 palavras por minuto (CHAFETS, 2006), uma velocidade que atrapalha e faz o som chegar aos ouvidos como murmúrio e ruído. 0 Guinness
Book cita que 0 campeão mundial em velocidade chegou a falar 637 palavras por minuto ${ }^{22}$, superando 0 antecessor que falava $586^{23}, 0$ que faz lembrar, às vezes, a narração esportiva no Brasil.

Mas é na vagarosa conversa intimista, ao pé $d o$ ouvido, que os facilitadores aconselham e medeiam conflitos. É por isso também que o programa religioso da Igreja Universal do Reino de Deus, transmitido nas madrugadas pela Rede Record no Brasil, chama-se apropriadamente de "Fala que Eu Te Escuto"24. É uma promessa magnânima. Com essa qualidade a tiracolo, as pessoas também estabelecem vínculos interculturais.

Naturalmente, escutar atentamente acarreta esforço para superar barreiras, como a ambiguidade e a vaguidade da prosa do orador. Tal empenho é sinal de boa vontade e cooperação, algo que gera reciprocidade e estimula a civilidade. A linguagem popularizou o efeito oposto na expressão "entrou por um ouvido e saiu pelo outro": é o que ocorre quando predominam a desatenção e a falta de conexão entre os interlocutores. A fórmula do bom ouvinte que decide escutar parece ser a da pessoa que interage com o falante e que lhe faz perguntas de forma interessada, as que são capazes de gerar novas ideias e as que desafiam sem hostilidade seus pressupostos (ZENGER \& FOLKMAN, 2016). Escutar com empatia é, em suma, saber dialogar (BURLEY-ALLEN, 1982). 
Conforme H. P. GRICE (1991), o falante que coopera com o ouvinte informa-o de forma clara, oferecendo-lhe nem mais nem menos informação que o necessário ao seu entendimento. Além do mais, a informação deve ser verdadeira e relevante para a compreensão. Naturalmente, tais princípios são frequentemente violados, algo fácil de ser observado na linguagem natural, a que é utilizada no dia a dia pelas pessoas.

Ocorre que há violações e obstáculos também no polo da audição. Elas podem ser geradas por barreiras físicas (sons concorrentes, por exemplo); por fatores linguísticos (a retórica do falante; o seu tom e timbre de voz; à vaguidade, ambiguidade e velocidade de sua prosa); por falta de contexto, algo que impede a decifração das implicaturas conversacionais; por dificuldades físicas (cansaço e estresse do ouvinte) e por emoções perturbadoras (raiva, medo e ansiedade, por exemplo). As barreiras cognitivas incluem 0 desinteresse, o pré-julgamento, as conclusões apressadas, as interrupções e reações a termos e rótulos abrasivos utilizados pelo falante. As barreiras culturais também afastam os interlocutores cujos valores e atitudes se distinguem fortemente. Em suma, quem não escuta bem não responde bem. Nesse tipo de ambiente predomina o ruído.

As barreiras psicológicas são as que maculam a empatia, uma das principais emoções ligadas à comunicação. Entre elas estão, por exemplo, 0 preconceito, o egocentrismo e o devaneio, algo que decorre em consequência da desatenção. Isso acontece porque o ouvinte pode processar, num minuto, entre 400 e 800 palavras, ou seja, cinco vezes mais conteúdo do que uma pessoa pode expressar oralmente. A diferença de ritmos permite ao ouvinte desviar e alocar uma parte de sua energia auditiva e cognitiva para outros pensamentos (HARGIE, 2011; p. 195).

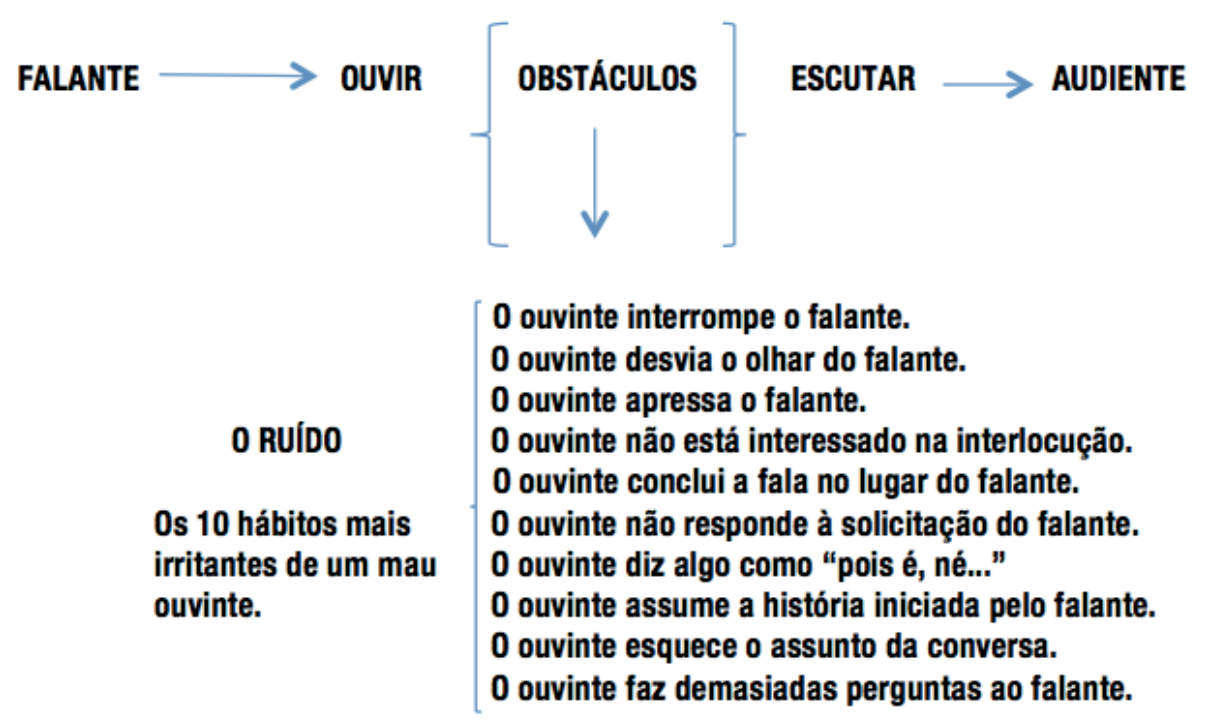


Como mencionado, as emoções acionadas pelo falante transformam quem ouve em audiente. 0 tema tem sido intensamente abordado no campo da psicologia da música, o que está interessado em entender o papel dos arranjos - seu ritmo, as escalas, a amplitude do som e a melodia - no acionamento de certos sentimentos do público, algo que serve também aos fins da terapia musical. Isso acontece porque 0 ouvinte espera certo tipo de tom, mas surge inesperadamente outro que 0 surpreende. É o caso da apogiatura ${ }^{25}$. A síncope produz efeito similar já que tal ritmo parece ser um distúrbio do fluxo regular do som (SLOBOBA, 2003) ${ }^{26}$. A verdade é que a diferença, qualquer diferença, tem esta virtude - ela chama a atenção do observador e gera nele sempre uma reação afetiva (WAINBERG, 2003).

\section{Estudo}

0 objetivo deste estudo é avaliar as habilidades cognitivas da escuta de 80 universitários do terceiro semestre da Faculdade de Comunicação da PUCRS, um dado que ajuda decifrar o perfil deste tipo de audiente. ${ }^{27} 0$ tema é relevante não só para ilustrar a abordagem teórica apresentada como também para documentar este que é um atributo típico do estudante. Ele gasta entre 42 e $53 \%$ do seu tempo escutando algo (geralmente a fala do professor). Cabe salientar também que $85 \%$ do que ele sabe depende desta habilidade (BARKER et. al, 1980; p.109).

Os universitários integrantes da amostra responderam ao Hurier Listening Profile, um questionário no qual o respondente classifica a qualidade de sua escuta a partir de 36 proposições ${ }^{28}$. Essa classificação é feita de 1 (quase nunca) a 5 (quase sempre) em cada uma das seis habilidades cognitivas propostas por este modelo desenvolvido por Judi Brownell (1995). A soma mede os pontos obtidos em cada habilidade de cada respondente (tabela 1). Por fim é calculada a média geral, a que caracteriza o perfil médio da amostra (tabela 2).

Os dados apresentados a seguir mostram que ESCUTAR é algo difícil para 6,25\% da amostra. Esse grau de dificuldade é o maior entre todas as seis categorias cognitivas examinadas. Na escala de 6 a 30 , eles revelam que essa habilidade é classificada pelos respondentes como RAZOÁVEL $(18,41)$. A interpretação é classificada como B0A (23). Já a compreensão é RAZ0ÁVEL com viés para BOA $(20,8)$. 0 estudante típico também considera B0A (24) sua habilidade de AVALIAÇÃO. Recordar é uma habilidade considerada RAZOÁVEL $(20,65)$ com viés para

25 É uma nota escrita um grau acima ou abaixo da nota que a sucede. Está fora de harmonia, como no início de Yesterday, cantada pelos Beatles.

$26 \mathrm{https} / / /$ www.youtube.com/watch?v=0d66ng6gaGw

27 Em 2014, o Brasil tinha próximo de oito milhões de estudantes matriculados em instituições de nível superior (MEC, 2016). 
BOA. Por fim, a habilidade RESPONDER é BOA

$(21,05)$. Esses resultados são apresentados a seguir também na escala de um a 24.

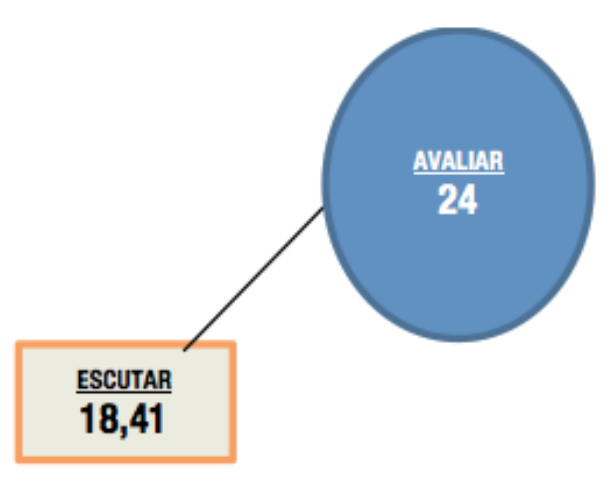

A habilidade com melhor desempenho categoria EXCELENTE - é AVALIAR (40,96\% dos respondentes). Enquanto a habilidade ESCUTAR dos universitários seja, como exposto, na média, somente RAZ0ÁVEL (algo que implica focar a atenção e selecionar os estímulos), e em quase 7\% dos casos seja FRACA, quase a metade da amostra declara que gosta de AVALIAR (julgar) o que escuta.

Tabela 1: As habilidades cognitivas da escuta

ESCUTAR

\begin{tabular}{|c|c|c|c|c|c|c|c|c|c|}
\hline Pontos & Frequência & Total & $\%$ & Frequência & Total & $\%$ & Frequência & Total & $\%$ \\
\hline 12 & 1 & 12 & 1,25 & - & - & - & - & - & - \\
\hline 13 & 1 & 13 & 1,25 & - & - & - & - & - & - \\
\hline 14 & 1 & 14 & 1,25 & - & - & - & - & - & - \\
\hline 15 & 2 & 30 & 2,5 & 1 & 15 & 0,81 & - & - & - \\
\hline 16 & 3 & 48 & 3,75 & 1 & 16 & 0,87 & 2 & 32 & 1,9 \\
\hline 17 & 5 & 85 & 6,25 & 2 & 34 & 1,84 & 1 & 17 & 1 \\
\hline 18 & 6 & 108 & 7,5 & 2 & 36 & 1,9 & 10 & 180 & 10,6 \\
\hline 19 & 7 & 133 & 8,75 & 12 & 228 & 12,38 & 11 & 209 & 12,3 \\
\hline 20 & 10 & 200 & 12,5 & 3 & 60 & 3,25 & 8 & 160 & 9.5 \\
\hline 21 & 11 & 231 & 13,75 & 1 & 21 & 1,14 & 6 & 126 & 7,5 \\
\hline 22 & 7 & 154 & 8,75 & 10 & 220 & 11,95 & 13 & 286 & 17 \\
\hline 23 & 5 & 115 & 6,25 & 8 & 184 & 9,99 & 9 & 207 & 12,3 \\
\hline 24 & 7 & 168 & 8,75 & 12 & 288 & 15,64 & 9 & 216 & 12,9 \\
\hline 25 & 5 & 125 & 6,25 & 9 & 225 & 12,22 & 5 & 125 & 7,4 \\
\hline 26 & 3 & 78 & 3,75 & 10 & 260 & 1,41 & 1 & 26 & 1,6 \\
\hline 27 & 3 & 81 & 3,75 & 3 & 81 & 4,40 & 3 & 81 & 4,8 \\
\hline 28 & - & - & - & 2 & 56 & 3,04 & - & - & \\
\hline 29 & 2 & 58 & 2,5 & 3 & 87 & 4,72 & - & - & \\
\hline 30 & - & - & - & 1 & 30 & 1,62 & 1 & 30 & \\
\hline Média & & 18,41 & & & 23 & & & 20,8 & \\
\hline
\end{tabular}


AVALIAR

RECORDAR

RESPONDER

\begin{tabular}{|c|c|c|c|c|c|c|c|c|c|}
\hline & Frequência & Total & $\%$ & Frequência & Total & $\%$ & Frequência & Total & $\begin{array}{l}\text { RP } \\
\%\end{array}$ \\
\hline 10 & - & - & - & 1 & 10 & 0.6 & - & - & - \\
\hline 11 & - & - & - & - & - & - & - & - & - \\
\hline 12 & - & - & - & - & - & - & - & - & - \\
\hline 13 & - & - & - & - & - & - & - & - & - \\
\hline 14 & - & - & - & - & - & - & - & - & - \\
\hline 15 & - & - & - & - & - & - & 1 & 15 & 0,9 \\
\hline 16 & - & - & - & 4 & 64 & 3,9 & 1 & 16 & 0,9 \\
\hline 17 & - & - & - & 10 & 170 & 10,4 & 5 & 85 & 5 \\
\hline 18 & - & - & - & 5 & 90 & 5,5 & 9 & 162 & 9,6 \\
\hline 19 & 5 & 95 & 4,95 & 12 & 228 & 13,9 & 9 & 171 & 10,2 \\
\hline 20 & 9 & 180 & 9,38 & 7 & 140 & 8,5 & 11 & 220 & 13 \\
\hline 21 & 5 & 105 & 5,47 & 7 & 147 & 8,9 & 8 & 168 & 9,8 \\
\hline 22 & 5 & 110 & 5,7 & 8 & 176 & 10,7 & 11 & 242 & 14,4 \\
\hline 23 & 14 & 322 & 16,8 & 7 & 161 & 9,8 & 10 & 230 & 19,5 \\
\hline 24 & 6 & 144 & 7,5 & 14 & 336 & 20,5 & 6 & 144 & 8,6 \\
\hline 25 & 7 & 175 & 9,12 & 1 & 25 & 1,5 & 5 & 125 & 7,4 \\
\hline 26 & 10 & 260 & 13,55 & 3 & 78 & 4,8 & 2 & 52 & 3,08 \\
\hline 27 & 11 & 297 & 15,48 & 1 & 27 & 1,7 & 2 & 54 & 3,2 \\
\hline 28 & 3 & 84 & 4,37 & - & - & - & - & - & - \\
\hline 29 & 4 & 116 & 6 & - & - & - & - & - & - \\
\hline 30 & 1 & 30 & 1,56 & - & - & - & - & - & - \\
\hline Média & - & 24 & - & - & 20,65 & - & - & 21,05 & - \\
\hline
\end{tabular}

Tabela 2: Resumo - as habilidades cognitivas da escuta.

\begin{tabular}{|c|c|c|c|c|c|c|}
\hline Ouvir & Interpretar & Compreender & Avaliar & Recordar & Responder & \\
\hline $\mathbf{1 8 , 4 1}$ & 23 & 20,8 & $\mathbf{2 4}$ & 20,65 & 21,05 & Escala de 6 a 30 \\
\hline $\mathbf{1 2 , 2 7}$ & 17 & 14,56 & $\mathbf{1 8}$ & 14,45 & 15 & Escala de um a 24 \\
\hline
\end{tabular}

10/15 pontos - 0 respondente percebe que tem problemas

$16 / 20$ pontos - 0 respondente classifica sua habilidade como adequada

$21 / 25$ pontos - 0 respondente classifica sua habilidade como boa

26/30 pontos - o respondente classifica sua habilidade como excelente
RAZOÁVEL

BOA

EXCELENTE 
Esse resultado documenta algo que a experiência prática de sala de aula mostra. Capturar e manter a atenção do aluno, a que deve estar fixada na exposição oral do professor, é missão difícil. Isso implica produzir, no polo da audição, uma frequência constante de estímulos, em especial os dotados com carga relevante de emoção. Esse desafio ocorre também noutras experiências de interlocução, nas quais o devaneio do público está sempre à espreita. É o caso, por exemplo, da cinematografia. 0 primeiro impacto, o que revela a trama, o herói e o vilão, é decisivo para mobilizar os sentidos da audiência. Depois, o mistério e, por fim, o desenlace sustenta a percepção de quem vive na sala de cinema um estado similar ao da hipnose.

0 público sempre ouve as narrativas com algum grau de apreensão. Isso também acontece no jornalismo, em que a boa notícia é, geralmente, a má notícia. 0 público é satisfeito nessa necessidade de desfrute emocional proporcionada pela vigilância ao meio ambiente. No caso, predominam 0 temor e a necessidade de controlálo diminuindo a incerteza. Também a informação audiovisual conquista a atenção, assegura 0 seu desfrute e permite às pessoas fazerem aquilo de que elas mais gostam: julgar os incidentes e os seus protagonistas.

Em resumo, o inquérito apresentado revela um dos principais desafios da docência. É necessário estimular a audição e, assim, controlar em medida significante a atenção, a compreensão, a análise e a síntese cognitiva do estudante. 0 devaneio é válvula de escape da qual se socorrem todos os públicos enfadados. Esse obstáculo, causado pelo desejo do ouvinte de obter o máximo benefício emocional com o menor desgaste cognitivo possível, demanda uma estratégia capaz de reverter 0 efeito do cansaço, do desinteresse e da dificuldade das pessoas em processar os estímulos, em especial, os mais complexos.

\section{Referências}

BARKER, Larry; Karen Gladney, Renee Edwards, Frances Holley \& Connie Gaines. An investigation of proportional time spent in various communication activities by college students'. Journal of Applied Communication Research v. 8, n. 2, 1980.

BEARD, D. \& BODIE, G. d. 'Listening research in the communication discipline.' In P. J. Gehrke \& M. Keith (eds). The unfinished conversation: 100 years of communication studies (pp. 207-233). New York: Routledge, 2014.

BICKFORD, Susan. The Dissonance of Democracy: Listening, Conflict, and Citizenship. Cornell University Press; 1996.

\section{BL00M, Benjamin S. Taxonomy of Educational}

Objectives. Allyn \& Bacon, Boston, MA. 1956.

BODIE, Graham D. 'Listening.' Oxford Research Encyclopedia of Communication. 2016.

BOMMEJLE, RICK. Listening Pays. HCI Printing \& Publishing. 2013.

BOSTROM, R.N. \& Bryant, C.L. 'Factors in the retention of information presented orally: The role of short-term listening.' Western Journal of Speech Communication, 44, 137-145. 1980.

BROADBENT, D.E. (1954). 'The role of auditory 
localization and memory span'. Journal of

Experimental Psychology 47 (3): 191-196, 1954.

BROWNELL, Judi. Listening: Attitudes, Principles, and Skills. Taylor \& Francis. 1995.

BURLEY-ALLEN, Madelyn. Listening the Forgotten

Skill. John Wiley \& sons. 1982.

CHAFETS, Zev. 'Ministers of Debate'. The New York

Times. 19/03/2009.

CHERRY, E. Colin, 'Some Experiments on the Recognition of Speech, with One and with Two Ears'. The Journal of the Acoustical Society of America. 25, (5): 975-79. 1953.

CODINA, Charlotte; Olivier Pascalis; Heidi A. Baseler, Alexandra T. Levine, David Buckley. 'Peripheral Visual Reaction Time Is Faster in Deaf Adults and British Sign Language Interpreters than in Hearing Adults'. Frontiers in psychology 8:50 • Fevereiro 2017.

COLLINS, D.S. Listening and Responding. NewDelhi: Cengage Learning. 2006.

CORNELLI, Gabriele. A Comunidade Pitagórica: Tipologia e Identidade. Hypnos, São Paulo, n. 27, 2011.

CURSI, Antonieta \& Belleli, Guglielmo. 'Cognitive and social consequences of exposure to emotional narratives: two studies on secondary social sharing of emotions.' Cognition and Emotion. 18 (7), p.881-900, 2004.

DEUTSCH, J.A.; Deutsch, D. Attention: Some

Theoretical Considerations.' Psychological

Review. 70 (I): 80-90, 1963.

DOBSON, Andrew. Listening for Democracy. Oxford University Press. 2014.

EMMERT, P. 'President's perspective.' ILA Listening Post, 56, 2-3. 1996.

FLYNN, J., Valikoski, T. \& Grau, J. 'Listening in the business context: Reviewing the state of research.'
International Journal of Listening, 22(2), 141-151. 2008 .

FROMM, Erich. The Art of Listening. Open Road Media. 2013.

GEARHART, C. C.; DENHAM, J. D. \& BODIE, G. D. 'Listening as a goal directed activity.' Western Journal of Speech, 78, p. 668-684. 2014.

GLENN, E. 'A content analysis of fifty definitions of listening.' Journal of the International Listening Association, 3, 1989.

GRANDJEAN, D., BANZIGER, T. \& Scherer, K.R. 'Intonation as an interface between language and affect.' Progress in Brain Research, 156, 235-268. 2006.

GRICE, H.P. 'Logic and conversation.' Studies in the way of words. New York: Harvard University, 1991.

HARGIE, Owen. Skilled Interpersonal Interaction: Research, Theory, and Practice. London: Routledge. 2011.

JANUSIK, Laura Ann. Researching listening from the inside out: the relationship between conversational listening and perceived communicative competence. Tese de Doutorado. Universidade de Maryland. 2004. . 'Building listening theory: The validating of the conversational listening span.' Communication Studies, 58, 139-156. 2007

JOHNSON, Susan M. 'Listening to the music: emotion as a natural part of systems theory.' Journal of

Systemic Therapies.v.17, n.2, 1998. pp. 1-17

KAHN, Charles H. Pitágoras e os Pitagóricos - uma breve história. Edições Loyola. 2001

KAHNEMAN, D. Attention and effort. Englewood Cliffs, NJ: Prentice-Hall. 1973,

LIMA, Ricardo Franco de. 'Compreendendo os Mecanismos Atencionais'. Ciências \& Cognição. v. 6, n.1 RJ. 2005, 
McGRAW, A. P.; Warren, C. 'Benign Violations'.

Psychological Science. 21 (8): pp. 1141-1149. 2010.

MEC. Mapa do Ensino Superior. 2016.

MENEZES, Carolina Baptista \& Dell'Aglio, Débora

Dalbosco. '0s efeitos da meditação à luz da

investigação científica em Psicologia: revisão de

literatura.' Psicol. cienc. prof. v.29 no.2 Brasília, 2009.

MENEZES, José Eugenio de 0. Cultura do ouvir: os vínculos sonoros na contemporaneidade. Revista

Líbero, ano IX, n.21, Junho de 2008.

MILLER, J.B.; DE WINSTANLEY, P.A. \& CAREY, P.

'Memory for conversation.' Memory, 4(6), 16-31. 1996.

MILLER, J.B., \& DE WINSTANLEY, P.A. 'The role of interpersonal competence in memory for conversation.' Personality and Social Psychology Bulletin, 28(1), 78- 89. 2002.

MORTON, J. \& Trehub, S. 'Children's understanding of emotion in speech.' Child Development, 72, p. 834-843. 2011.

NICHOLS, Ralph G. 'Listening is 10 part skill.' Nation's Business. Julho de 1957.

\& Leonard A. Stevens. 'Listening to People.'

Harvard Business Review v. 35, n.5, pp. 85-92.

Setembro de 1957.

PELL, Marc D., Jaywant, Abhishek; Monetta, Laura

\& Kotz, Sonja A. 'Emotional Speech processing:

Disentangling the effects of prosody and semantic

cues.' Cognition and Emotion. 25 (5), p. 834-853, 2011.

PURDY, M. 'What is listening?' In M. Purdy \& D. Borisoff (Eds.), Listening in everyday life: A personal and professional approach. (pp. 1-20). Lanham, MD: University Press of America. 1996.

RAINA, Reeta; ROEBUCK, Deborah Britt \& LEE, CHENG EAN (Catherine). An Exploratory Study of Listening Skills of Professionals across Different
Cultures.' World Review of Business Research v. 4. n. 3. pp. 1-13. Outubro, 2014.

ROEBUCK, Deborah; Bell, Reginald L; Raina; Lee, Reeta Cheng Ean (Catherine). 'The effects of home country, gender, and position on listening behaviors.' Journal of Organizational Culture, Communications and Conflict. v. 19, n.2. 2015.

SECOM. Pesquisa Brasileira de Mídia. 2015.

SL0B0BA, John. 'Show me Emotion.' New Scientist. V. 180, n. 2423. 11/29/2003.

STEIL, LYMAN K., Larry L. Barker \& Kittie W. Watson. Effective Listening: Key to your success. 1983.

TREISMAN, Anne M. 'Strategies and models of selective attention.' Psychological Review. 76 (3): 282-299,1969.

TURKLE, Shery. Reclaiming Conversation. Peguin Books. 2016

WAINBERG, Jacques A. 'A Voz de Deus - A Persuasão Audiovisual de Um Povo'. INTERCOM - Revista Brasileira de Comunicação, v. 15, n.2, p. 144-166, 1992.

Turismo e Comunicação: a indústria da diferença. São Paulo: Contexto, 2003.

Línguas Ferinas. Porto Alegre: Edipucrs. 2010.

WILLIAMS, J. R. 'Guidelines for the use of multimedia in instruction.' Proceedings of the Human Factors and Ergonomics Society 42nd Annual Meeting, 1447-1451, 1998.

WILSON, Deirdre. \& Wharton, Tim. 'Relevance and Prosody.' Journal of Pragmatics, 38, p. 1559-1579. 2006.

WOLVIN, A. 'Culture as a listening variable.'

International Listening Association Summer

Conference, Toronto, Canada. 1987.

WOLVIN, A. D., \& Coakley, C. G. 'A listening taxonomy.' 
In A. D. Wolvin \& C. G. Coakley (Eds.), Perspectives on listening (pp. 15-22). Norwood, NJ: Ablex. 1993.

VAN ALPHEN, Markus. Observational Listening.

AuthorHouse. 2016.

ZENGER, Jack \& FOLKMAN, Joseph. What Great

Listeners Actually Do. 2016. [https://hbr.org/2016/07/

what-great-listeners-actually-do] 


\begin{tabular}{|c|c|}
\hline Communication and Listening & Comunicación y escucha \\
\hline $\begin{array}{l}\text { Abstract } \\
\text { This is a theoretical and empirical study on listening, } \\
\text { a skill that implies the cognitive and emotional } \\
\text { attributes of the listener. Theoretical review shows } \\
\text { its main elements and challenges. It concerns all } \\
\text { kinds of interlocutions, such as conversation and } \\
\text { media tune, for example. The Hurier Listening Profile } \\
\text { questionnaire is applied to } 80 \text { Brazilian university } \\
\text { students. The data collected show that listening } \\
\text { faces the greatest difficulties among the six cognitive } \\
\text { attributes. This study also highlights the role of } \\
\text { emotions in stimulating the attention of the audience. } \\
\text { Keywords } \\
\text { Listening. Emotion. Cognition. }\end{array}$ & $\begin{array}{l}\text { Resumen } \\
\text { Este es un estudio teórico y empírico sobre la } \\
\text { escucha, una habilidad que implica en atributos } \\
\text { cognitivos y emocionales del oyente. La reseña } \\
\text { teórica muestra sus principales elementos y } \\
\text { desafíos. Se refiere a todo tipo de interlocución, } \\
\text { como la conversación y la sintonía de los medios } \\
\text { de comunicación, por ejemplo. Se aplica aquí } \\
\text { el cuestionario Hurier Listening Profile a } 80 \\
\text { universitarios brasileños. Los datos levantados } \\
\text { muestran que la escucha enfrenta las mayores } \\
\text { dificultades entre los seis atributos cognitivos } \\
\text { involucrados en la escucha. El estudio destaca } \\
\text { también el papel de las emociones en la estimulación } \\
\text { de la atención de la audiencia. } \\
\text { Palabras-clave } \\
\text { Escucha. Emoción. Cognición. }\end{array}$ \\
\hline
\end{tabular}




\section{Expediente}

A revista E-Compós é a publicação científica em formato eletrônico da Associação Nacional dos Programas de Pós-Graduação em Comunicação (Compós). Lançada em 2004, tem como principal finalidade difundir a produção acadêmica de pesquisadores da área de Comunicação, inseridos em instituições do Brasil e do exterior.

\section{E-COMPÓS I www.e-compos.org.br I E-ISSN 1808-2599}

Revista da Associação Nacional dos Programas de Pós-Graduação em Comunicação. Brasília, v.21, n.1, jan./abr. 2018. A identificação das edições, a partir de 2008 , passa a ser volume anual com três números. Indexada por Latindex I www.latindex.unam.mx

\section{CONSELHO EDITORIAL}

Ada Cristina Machado Silveira, Universidade Federal de Santa Maria, Brasil Alda Cristina Silva da Costa, Universidade Federal do Pará, Brasil Alfredo Luiz Paes de Oliveira Suppia, Universidade Estadual de Campinas, Brasil Ana Regina Barros Rego Leal, Universidade Federal do Piauí, Brasil Ana Carolina Rocha Pessôa Temer, Universidade Federal de Goiás, Brasil André Luiz Martins Lemos, Universidade Federal da Bahia, Brasil Angela Cristina Salgueiro Marques, Universidade Federal de Minas Gerais, Brasil Ângela Freire Prysthon, Universidade Federal de Pernambuco, Brasil Antonio Carlos Hohlfeldt, Pontifícia Universidade Católica do Rio Grande do Sul, Brasil Arthur Ituassu, Pontifícia Universidade Católica do Rio de Janeiro, Brasil

Bruno Campanella, Universidade Federal Fluminense, Brasil

Cláudio Novaes Pinto Coelho, Faculdade Cásper Líbero, Brasil Cárlida Emerim, Universidade Federal de Santa Catarina, Brasil Carlos Eduardo Franciscato, Universidade Federal de Sergipe, Brasil Danilo Rothberg, Universidade Estadual Paulista, Brasil Denise Tavares da Silva, Universidade Federal Fluminense, Brasil Diógenes Lycarião, Universidade Federal do Ceará, Brasil Eduardo Vicente, Universidade de São Paulo, Brasil Eliza Bachega Casadei, Escola Superior de Propaganda e Marketing - SP, Brasil Eneus Trindade, Universidade de São Paulo, Brasil

Erick Felinto de Oliveira, Universidade do Estado do Rio de Janeiro, Brasil Erly Vieira Júnior, Universidade Federal do Espírito Santo, Brasil Francisco de Assis, FIAM-FAAM Centro Universitário, Brasi

Francisco Elinaldo Teixeira, Universidade Estadual de Campinas, Brasi Francisco Gilson R. Pôrto Jr., Universidade Federal do Tocantins, Brasil Frederico de Mello Brandão Tavares, Universidade Federal de Ouro Preto, Brasil Gabriela Reinaldo, Universidade Federal do Ceará, Brasil Gilson Vieira Monteiro, Universidade Federal do Amazonas, Brasil Gustavo Daudt Fischer, Universidade do Vale do Rio dos Sinos, Brasi Itania Maria Mota Gomes, Universidade Federal da Bahia, Brasil Jiani Adriana Bonin, Universidade do Vale do Rio dos Sinos, Brasil José Afonso da Silva Junior, Universidade Federal de Pernambuco, Brasil José Luiz Aidar Prado, Pontifícia Universidade Católica de São Paulo, Brasil Josette Maria Monzani, Universidade Federal de São Carlos, Brasi Juçara Gorski Brittes, Universidade Federal de Ouro Preto, Brasil
Juliana Freire Gutmann, Universidade Federal da Bahia, Brasil Laura Loguercio Cánepa, Universidade Anhembi Morumbi, Brasil Leonel Azevedo de Aguiar, Pontifícia Universidade Católica do Rio de Janeiro, Brasil Letícia Cantarela Matheus, Universidade do Estado do Rio de Janeiro, Brasil Luciana Coutinho Souza, Universidade de Sorocaba, Brasil Maria Ataide Malcher, Universidade Federal do Pará, Brasil Maria Elisabete Antonioli, Escola Superior de Propaganda e Marketing - SP, Brasil Maria das Graças Pinto Coelho, Universidade Federal do Rio Grande do Norte, Brasil Marialva Carlos Barbosa, Universidade Federal do Rio de Janeiro, Brasil Marcel Vieira Barreto Silva, Universidade Federal da Paraíba, Brasil Marcia Tondato, Escola Superior de Propaganda e Marketing, Brasil Marli Santos, Universidade Metodista de São Paulo, Brasil

Márcio Souza Gonçalves, Universidade do Estado do Rio de Janeiro, Brasil Mauricio Mario Monteiro, Universidade Anhembi Morumbi, Brasil Mayka Castellano, Universidade Federal Fluminense, Brasil

Mozahir Salomão Bruck, Pontifícia Universidade Católica de Minas Gerais, Brasil Nisia Martins Rosario, Universidade Federal do Rio Grande do Sul, Brasil Paolo Demuru, Universidade Paulista, Brasil

Paula Melani Rocha, Universidade Estadual de Ponta Grossa, Brasil Potiguara Mendes Silveira Jr, Universidade Federal de Juiz de Fora, Brasil Priscila Ferreira Perazzo, Universidade Municipal de São Caetano do Sul, Brasil Rafael Cardoso Sampaio, Universidade Federal do Paraná, Brasil Rafael Tassi Teixeira, Universidade Tuiuti do Paraná, Brasil Regiane Lucas Garcês, Universidade Federal de Minas Gerais, Brasil Regiane Regina Ribeiro, Universidade Federal do Paraná, Brasil Renata Pitombo Cidreira, Universidade Federal do Recôncavo da Bahia, Brasil Renato Essenfelder, Escola Superior de Propaganda e Marketing, Brasil Roberto Elísio dos Santos, Universidade Municipal de São Caetano do Sul, Brasil Rodolfo Rorato Londero, Universidade Estadual de Londrina, Brasil Roseli Figaro, Universidade de São Paulo, Brasil

Simone Maria Andrade Pereira de Sá, Universidade Federal Fluminense, Brasil Sofia Cavalcanti Zanforlin, Universidade Católica de Brasília, Brasil Sônia Caldas Pessoa, Universidade Federal de Minas Gerais, Brasil Tatiana Oliveira Siciliano, Pontifícia Universidade Católica do Rio de Janeiro, Brasil Thaïs de Mendonça Jorge, Universidade de Brasília, Brasil

Valquiria Michela John, Universidade Federal do Paraná, Brasil

\section{CONSELHO CIENTÍFICO}

Cristiane Freitas Gutfreind, Pontifícia Universidade Católica do Rio Grande do Sul, Brasil | Eduardo Antônio de Jesus, Universidade Federal de Minhas Gerais, Brasil I Eduardo Morettin, Universidade de São Paulo, Brasil I Irene de Araújo Machado, Universidade de São Paulo, Brasil I Miriam de Souza Rossini, Universidade Federal do Rio Grande do Sul, Brasil

\section{COMISSÃO EDITORIAL}

Igor Pinto Sacramento, Universidade Federal do Rio de Janeiro, Brasil I Kelly Cristina de Souza Prudencio, Universidade Federal do Paraná, Brasil I Osmar Gonçalves dos Reis Filho, Universidade Federal do Ceará, Brasil I Rafael Grohmann, Faculdade Cásper Líbero, Brasil I Thaiane Moreira de Oliveira, Universidade Federal Fluminense, Brasil (editores associados)

\section{CONSULTORES AD HOC}

Afonso de Albuquerque, Universidade Federal Fluminense, Brasil I Cláudia Lago, Universidade de São Paulo, Brasil I Cesar Baio Santos, Universidade Federal do Ceará, Brasil I Eduardo Pellanda, Pontifícia Universidade Católica do Rio Grande do Sul, Brasi | Francisco Rüdiger, Pontifícia Universidade Católica do Rio Grande do Sul, Brasil | Karina Woitowicz, Universidade Estadual de Ponta Grossa, Brasil I Luis Mauro Sa Martino, Faculdade Cásper Líbero, Brasil I Norval Baitello Jr, Pontifícia Universidade Católica de São Paulo, Brasil | Pedro Guimarães, Universidade de Campinas, Brasil

\section{EQUIPE TÉCNICA}

ASSISTENTES EDITORIAIS Márcio Zanetti Negrini e Melina Santos | REVISÃO DE TEXTOS Fátima Áli | EDITORAÇÃO ELETRÔNICA Roka Estúdio
COMPÓS I www.compos.org.br

Associação Nacional dos Programas de Pós-Graduação em Comunicação

Presidente

Marco Roxo

Programa de Pós-Graduação em Comunicação - UFF marcos-roxo@uol.com.br

Vice-Presidente

Isaltina Gomes

Programa de Pós-Graduação em Comunicação - UFPE

isaltina@gmail.com

Secretária-Geral

Gisela Castro

Programa de Pós-Graduação em Comunicação

e Práticas de Consumo - ESPM

castro.gisela@gmail.com

CONTATO I revistaecompos@gmail.com 